\title{
Desarrollo de Convertidores Electrónicos Bidireccionales de Potencia para Sistemas de Generación de Energías Renovables usando Control Predictivo
}

\author{
Juan F. Tisza C \\ Universidad Nacional de Ingeniería, Av. Túpac Amaru 210, Rímac, Lima, Perú \\ Recibido el 4 de diciembre del 2018, Aceptado el 27 de diciembre de 2018
}

DOI: https://doi.org/10.33017/RevECIPeru2018.0018/

\section{Resumen}

Este trabajo se desarrolla un convertidor electrónico bidireccional de potencia eléctrica AC/DC y se evalúan sus indicadores de rendimiento, todo este estudio en un contexto en el cual se busca incrementar la generación de fuentes de energía renovables en la matriz energética del Perú, en especial las fuentes solar y eólica. Actualmente es de particular importancia la consolidación de la generación de energía eléctrica distribuida [1].En este escenario los sistemas de conversión bilateral de energías DC y AC [5] son de gran importancia ,Se busca tener un comportamiento eficiente y con técnicas de control avanzadas que permitan reducir las distorsiones de armónicos, así como también tener la robustez ante perturbaciones y minimizar las eventuales inestabilidades que se produzcan al conectarse a las redes públicas de distribución eléctrica [2],(en forma cuantificada el objetivo es tener menos del $2 \%$ en THD, en la variable de interés). El desarrollo se realiza mediante la aplicación del Control Predictivo Basado en Modelos en su versión de aplicación llamada tipo Conjunto Finito de Conmutaciones ("Finite Commutation Set" FCS), así las unidades de control de la etapa de potencia son implementadas con control MPC-FCS. Se muestran los principales resultados obtenidos mediante herramientas de simulación (Matlab y PSIM) aplicados a estos sistemas electrónicos de potencia (Se establecen como objetivos de la investigación conseguir eficiencias eléctricas por encima del 98\%). En este estudio se tiene muy en cuenta la medición de la magnitud y la dirección del flujo de potencia y energía eléctrica entre las fuentes de generación, la red, la carga y los almacenadores de energía. Se presentan los resultados más significativos que muestran los resultados y la metodología que en definitiva tiene un mejor comportamiento que los implementados con los métodos ampliamente usados como el SVPWM.

Descriptores: Energías renovables, generación distribuida, convertidores electrónicos de Potencia, MPC-FCS, THD.

\section{Abstract}

This work develops a bidirectional electronic converter of $A C / D C$ electric power and evaluates its performance indicators, all this study in a context in which it seeks to increase the generation of renewable energy sources in the energy matrix of Peru, especially solar and wind sources. In this scenario, the bilateral conversion systems of DC and AC energies [5] are of great importance, we seek to have an efficient behavior and with advanced control techniques that allow us to reduce harmonic distortions, as well as to have the robustness before disturbances and minimize eventual instabilities that occur when connecting to the public electric distribution networks [2], (in a quantified way, the objective is to have less than $2 \%$ in THD, in the variable of interest). The development is done through the application of Predictive Control Based on Models in its version of application called Finite Commutation Set ("Finite Commutation Set" FCS), so the control units of the power stage are implemented with MPC-FCS control. The main results obtained using simulation tools (Matlab and PSIM) applied to these electronic power systems are shown (research objectives are established to achieve electrical efficiencies above 98\%). This study takes into account the measurement of the magnitude and direction of the flow of power and electrical energy between the generation sources, the grid, the load and the energy storers. 
The most significant results are presented, which show the results and the methodology, which, in short, performs better than those do, implemented with widely used methods such as SVPWM.

Keywords: Renewable energies, distributed generation, electronic power converters, MPC- FCS, THD.

\section{Introducción}

A diferencia de las grandes plantas centralizadas de combustible fósiles, como las termoeléctricas o las turbinas de vapor, las fuentes de energía renovable tienden a tener una arquitectura distribuida. Además de la conocida generación limpia se tienen sistemas de control mejorados que toman en cuenta consideraciones como los pronósticos climáticos, que toma en cuenta incertidumbres para robustecer el sistema. La Fig. 1 muestra de manera gráfica los sistemas de energía eléctrica tradicional y de próxima generación. Según [6], los sistemas de energía distribuidos poseen las ventajas de tener un mayor grado de distribuidos poseen las ventajas de tener un mayor grado de confiabilidad, de ser escalables de manera relativamente más sencilla que los sistemas convencionales y pueden ser flexiblemente controlados.
Entonces, aparece el concepto de una micro-red, que se trata de un sistema con varias fuentes de generación distintas que pueden ser controlados de manera individual en un nivel de control inferior y en conjunto en un nivel superior de control [7]. Usualmente, todas las redes individuales (unidades de generación distribuidas) se unen a un bus de energía. Este bus, lleva la energía hasta las cargas, y se puede realizar una gestión óptima de la energía, permite sacar de operación cualquiera de ellas sin afectar el desempeño del sistema global. Entonces, se puede definir a una micro-red inteligente como un sistema de generación bidireccional, que permite la distribución de electricidad, tanto desde los proveedores hasta los consumidores, como la venta de electricidad por parte de los segundos.

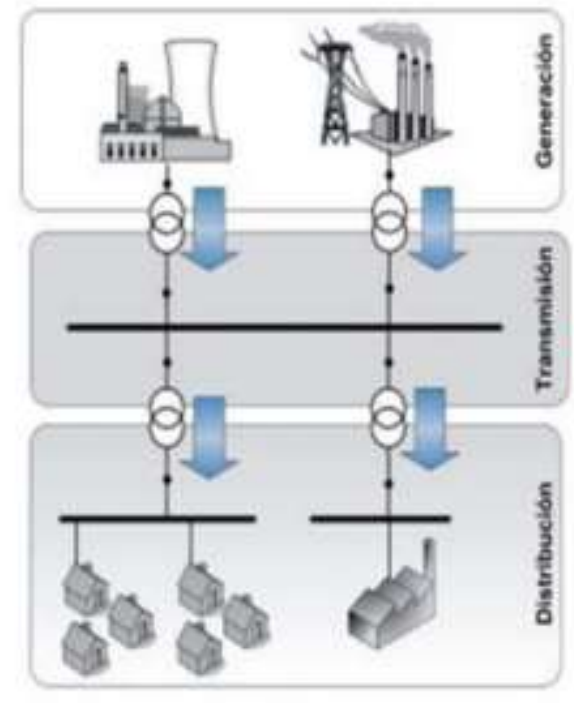

a

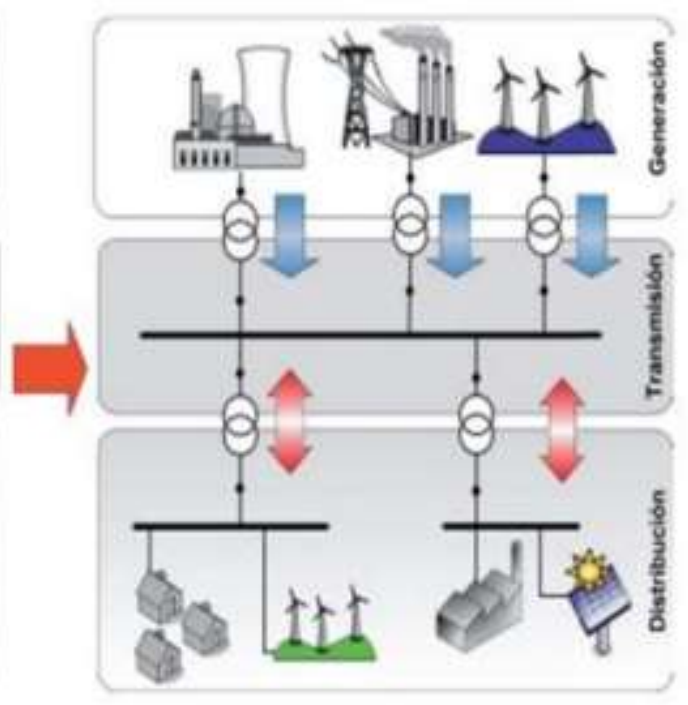

b

Figura 1: a) Sistema de generación energética tradicional y b) Sistema de generación distribuida con micro redes.

Los convertidores de energía eléctrica requeridos para esta forma de administración del suministro eléctrico requieren características como la bidireccionalidad, baja distorsión total de armónicos (THD), lo que hace que estos sistemas sean más versátiles para variados tipos de generación como sucede cuando usamos fuentes renovables.
En este trabajo diseñamos y probamos un convertidor bidireccional que es una solución para este requerimiento y se escoge el sistema de control MPC-FCS, pues este tipo de tecnología de control nos conduce a un diseño optimizado y con una mínima complejidad en la comprensión e implementación del sistema de control. 


\section{Metodología}

El diseño del convertidor de potencia bidireccional sigue 4 etapas que se describen a continuación:

La primera etapa es la selección de la topología del convertidor considerando aspectos circuitales que deben seleccionarse, de acuerdo a criterios operativos y económicos.

La segunda etapa se plantea las características específicas que establecemos para la aplicación del control MPC-FCS a la topología seleccionada, a partir de esa evaluación se implementa el algoritmo que se ejecutara con las herramientas que disponemos. En este trabajo utilizamos Matlab y Simulink.

La tercera etapa lo constituye la etapa de implementación a nivel de simulación del funcionamiento y el cálculo de las características del comportamiento, las mismas que serán utilizadas en la evaluación comparativa con otras alternativas de solución, en este trabajo calculamos el valor de la distorsión (THD) y la alternativa que utilizamos para la comparación el control SVPWM (Sine
Voltage Pulse Wight Modulation). La cuarta etapa considera la incorporación de características reales de los dispositivos electrónicos utilizados a partir de sus hojas de datos técnicos correspondientes, asi como las restricciones que se deben considerar en el sistema real, para finalmente recalcular la simulación y los parámetros que muestran la optimización y el nivel de eficacia de la conversión.

\subsection{Selección de Topología del convertidor para el diseño de Control predictivo FCS-MPC.}

Topología del inversor que se utiliza en este trabajo es mostrado en la figura 2 , se ha establecido que la etapa de potencia corresponda a una característica trifásica, el cual hace uso de los IGBT's como dispositivos de conmutación. En cada brazo solo 1 IGBT debe de conducir, por lo tanto, deben de estar complementados en su conmutación. En consecuencia, se tiene 8 estados de conmutación posibles. En la figura 3 se muestra la etapa de potencia y un primer modelamiento en su versión más simple para el conmutador en este caso los IGBTs y se incorpora la carga, se puede considerar integrados las características de la carga propiamente con un filtro inductivo resistivo.

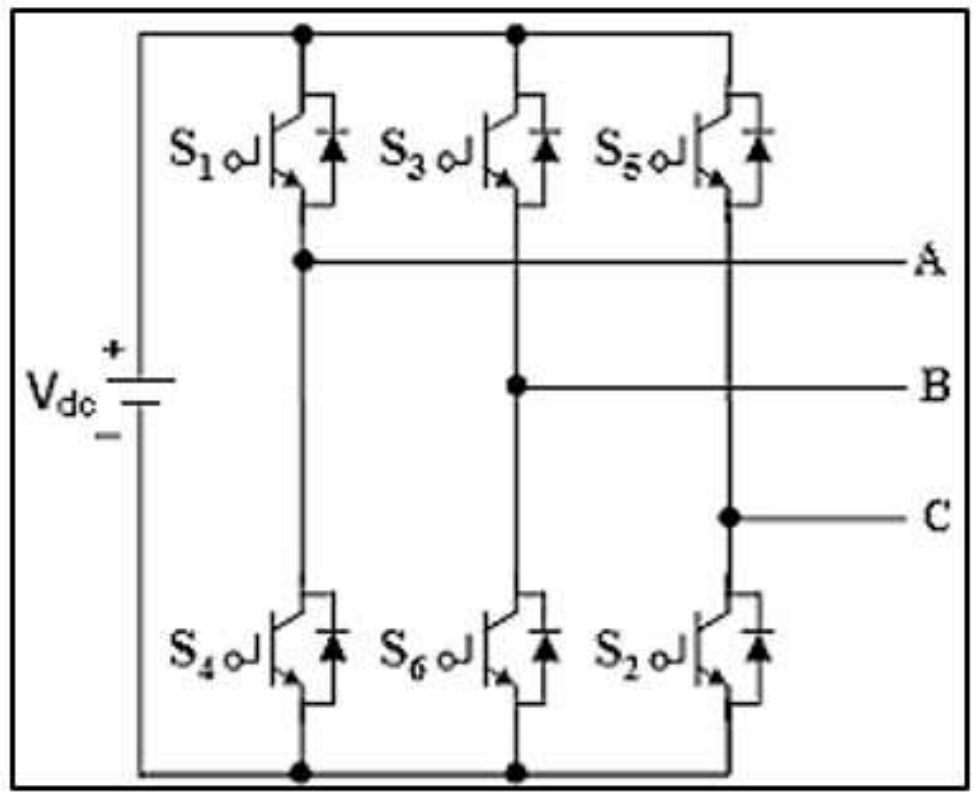

Figura 2: Topología circuital de la etapa de potencia del convertidor

\subsection{Funcionamiento de Inversor, Características Específicas y Relaciones para el Diseño}

El modelo para el FCS-MPC se obtiene analizando la dinámica en la carga. El voltaje en la carga sigue la siguiente ecuación:

$$
v=R i+L \frac{d i}{d t}
$$

En donde $R$ y $L$ son la carga resistiva e inductiva respectivamente, i es la corriente en la carga y $v$ es el voltaje generado por el inversor. Usando el 
método de Euler, la corriente en la carga se puede aproximar en tiempo discreto como:

$$
\frac{d i}{d t}=\frac{i(k+1)-i(k)}{T_{s}}
$$

De la ecuación 1 y 2 podemos obtener el modelo que prediga la corriente en el instante $\mathbf{k}+\mathbf{1}$ dado un voltaje generado en la salida del inversor.

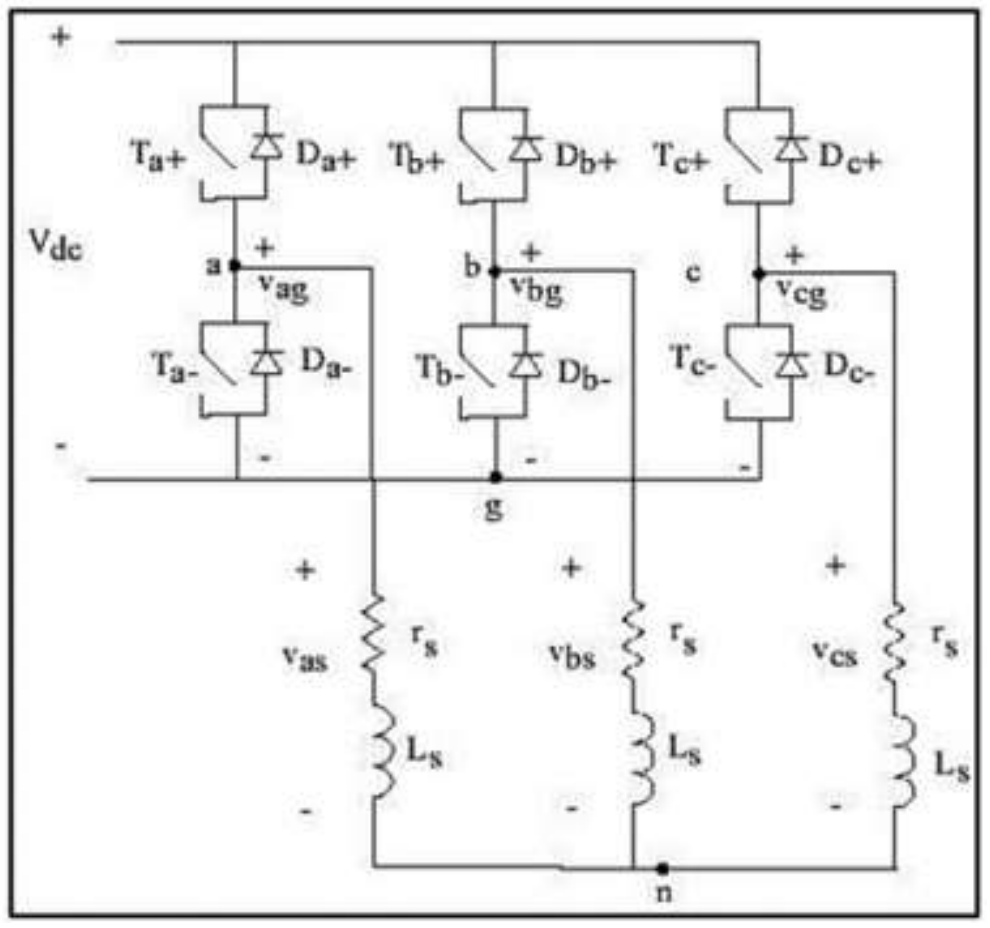

Figura 3: Inversor con el modelo elemental para el IGBT e incorporada el circuito de carga.

$$
i^{\prime}(k+1)=\left(1-\frac{R T_{S}}{L}\right) i(k)+\frac{T_{S}}{L} v(k)
$$

En donde $\mathrm{k}$ es el instante presente, $\mathrm{k}+1$ el instante futuro/predicho, T_S es el periodo de muestreo.

Para encontrar el estado de conmutación $S(k)$ optimo se define una función de costo para cada voltaje de salida generado por el inversor. El estado de conmutación óptimo es aplicado al inversor en el siguiente periodo de muestreo.

La función costo que se busca minimizar es la diferencia entre la corriente predicha y la corriente de referencia en la carga en el instante $k+1$. Generalmente se elige un periodo de muestreo suficientemente pequeño de modo que la corriente de referencia se mantiene constante en el siguiente instante. En consecuencia, la función de coste se define como:

$$
J(k+1)=\left|i^{\prime}(k+1)-i_{r e f}(k)\right|
$$

En donde $\boldsymbol{i}_{\text {ref }}$ es la corriente que se dará seguimiento. La función de coste $\boldsymbol{J}$ se calcula para todos los estados de conmutación $\mathrm{S}(\mathrm{k})$ y se escoge el estado de conmutación que produzca $J=\min (J)$.

\subsection{Implementación, Simulación del inversor}

Desarrollamos el circuito en MATLAB® para ser más específico usamos Simulink® para el esquema de los componentes. En el bloque Fcn desarrollamos el código que simboliza el control para que el inversor funcione.

\subsection{Funcionamiento de Rectificador, Características específicas y relaciones para el diseño}

Utilizando la teoría convencional de los rectificadores trifásicos de onda completa, donde los dispositivos de conmutación son IGBTs.

En un análisis específico para ángulos de disparo menor de $60^{\circ},\left(\alpha \leq 60^{\circ}\right)$, se obtiene para la tensión DC en la carga el valor siguiente:

$$
V_{d c}=\frac{3 V_{m}}{\pi}\left[1+\frac{1}{2} \cos (\alpha)-\frac{\sqrt{3}}{2} \operatorname{sen}(\alpha)\right]
$$


Tabla 1: Conjunto de conmutaciones posibles, para el inversor.

\begin{tabular}{|l|l|l|l|r|r|r|}
\hline Vector & Leg a & Leg b & Leg c & $\boldsymbol{V}_{\boldsymbol{a n}}$ & $\boldsymbol{V}_{\boldsymbol{b n}}$ & $\boldsymbol{V}_{\boldsymbol{c n}}$ \\
\hline $\boldsymbol{V}_{\mathbf{0}}$ & 0 & 0 & 0 & 0 & 0 & 0 \\
\hline $\boldsymbol{V}_{\mathbf{1}}$ & 0 & 0 & 1 & $-E / 3$ & $-E / 3$ & $2 E / 3$ \\
\hline $\boldsymbol{V}_{\mathbf{2}}$ & 0 & 1 & 0 & $-E / 3$ & $2 E / 3$ & $-E / 3$ \\
\hline $\boldsymbol{V}_{\mathbf{3}}$ & 0 & 1 & 1 & $-2 E / 3$ & $E / 3$ & $E / 3$ \\
\hline $\boldsymbol{V}_{\mathbf{4}}$ & 1 & 0 & 0 & $2 E / 3$ & $-E / 3$ & $-E / 3$ \\
\hline $\boldsymbol{V}_{\mathbf{5}}$ & 1 & 0 & 1 & $E / 3$ & $-2 E / 3$ & $E / 3$ \\
\hline $\boldsymbol{V}_{\mathbf{6}}$ & 1 & 1 & 0 & $E / 3$ & $E / 3$ & $-2 E / 3$ \\
\hline $\boldsymbol{V}_{\mathbf{7}}$ & 1 & 1 & 1 & 0 & 0 & 0 \\
\hline
\end{tabular}

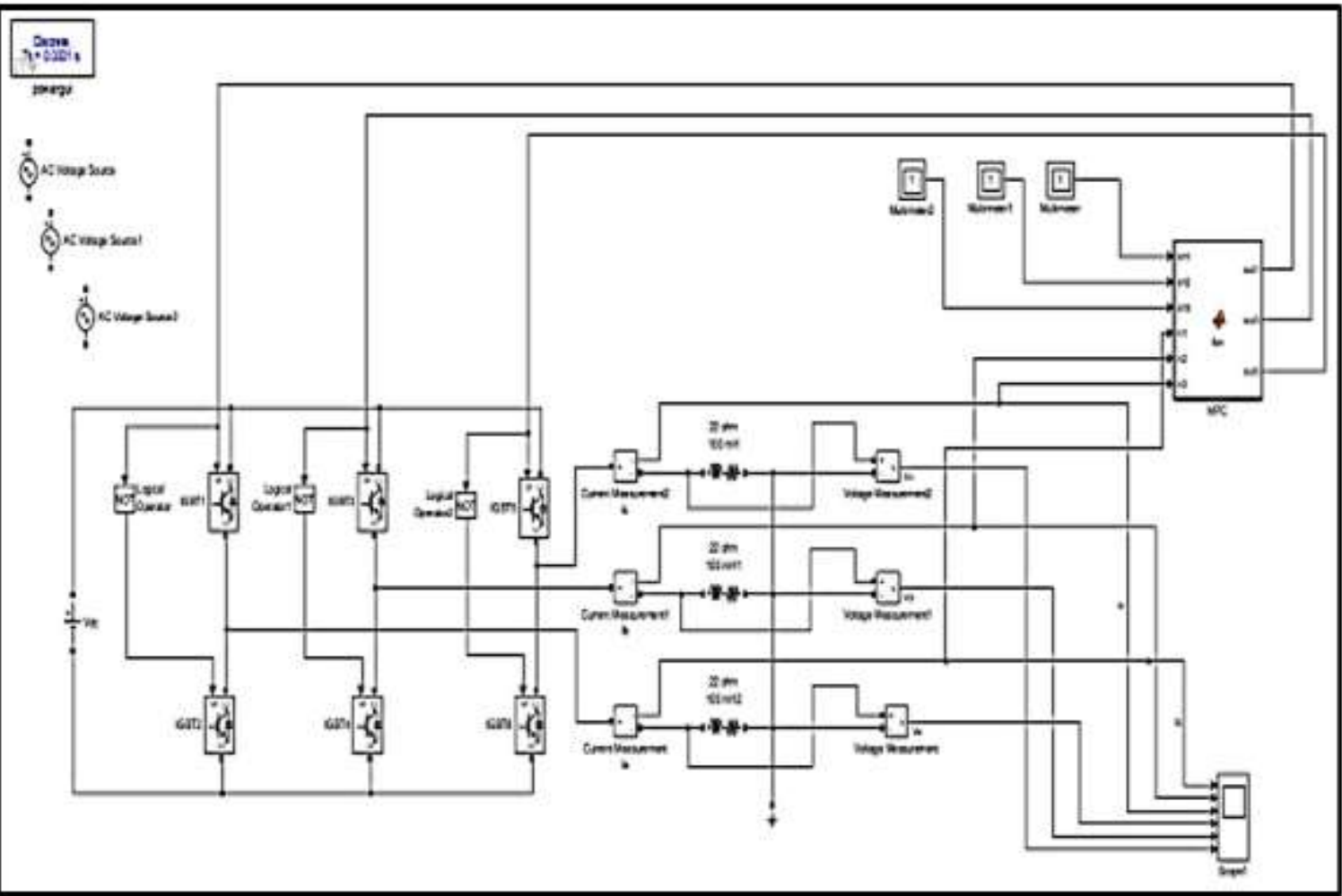

Figura 4: Esquema circuital utilizado para el inversor.

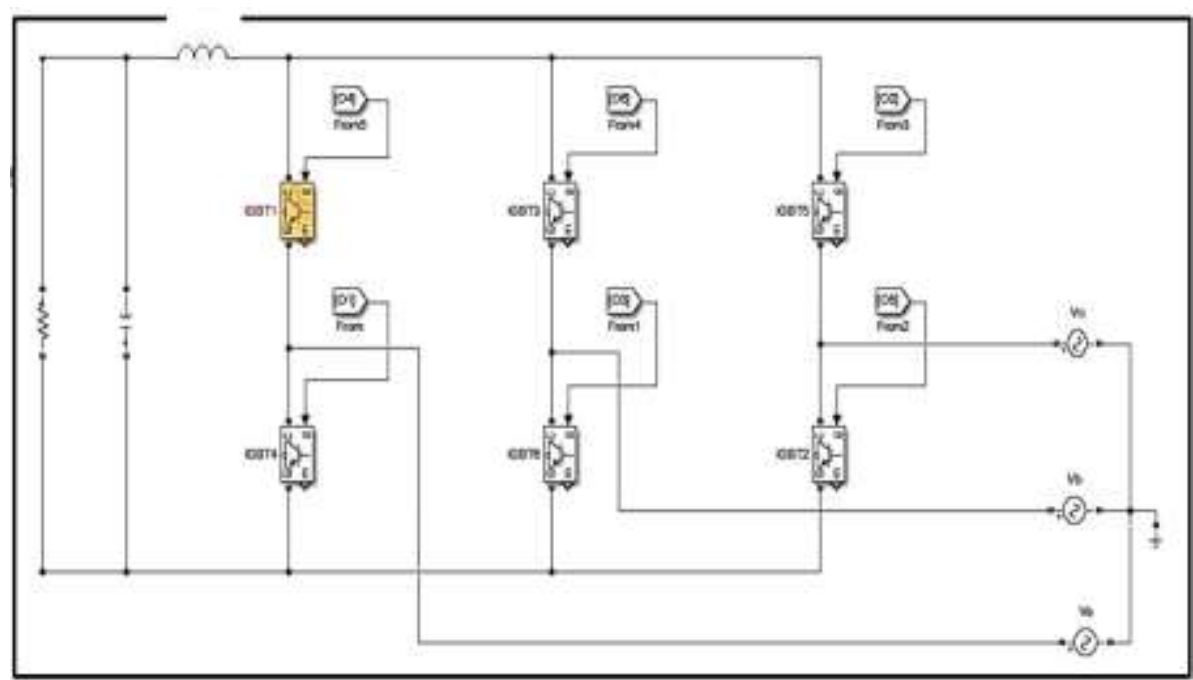


Figura 5: El Convertidor trifásico en su funcionamiento como rectificador.

Para el caso que estamos considerando tenemos la evaluación para el ángulo de disparo de $0^{\circ}$, pues el ángulo de disparo se mide a partir del instante en que la tensión de fase Va tiene un ángulo de $30^{\circ}$, se desarrolla en MATLAB® y Simulink ${ }^{\circledR}$ el circuito simulado, mostrado en la figura 6, En el bloque Fcn desarrollamos el código de control para que el rectificador funcione. En la tabla 2 se presenta la secuencia de conmutación de los IGBTs.

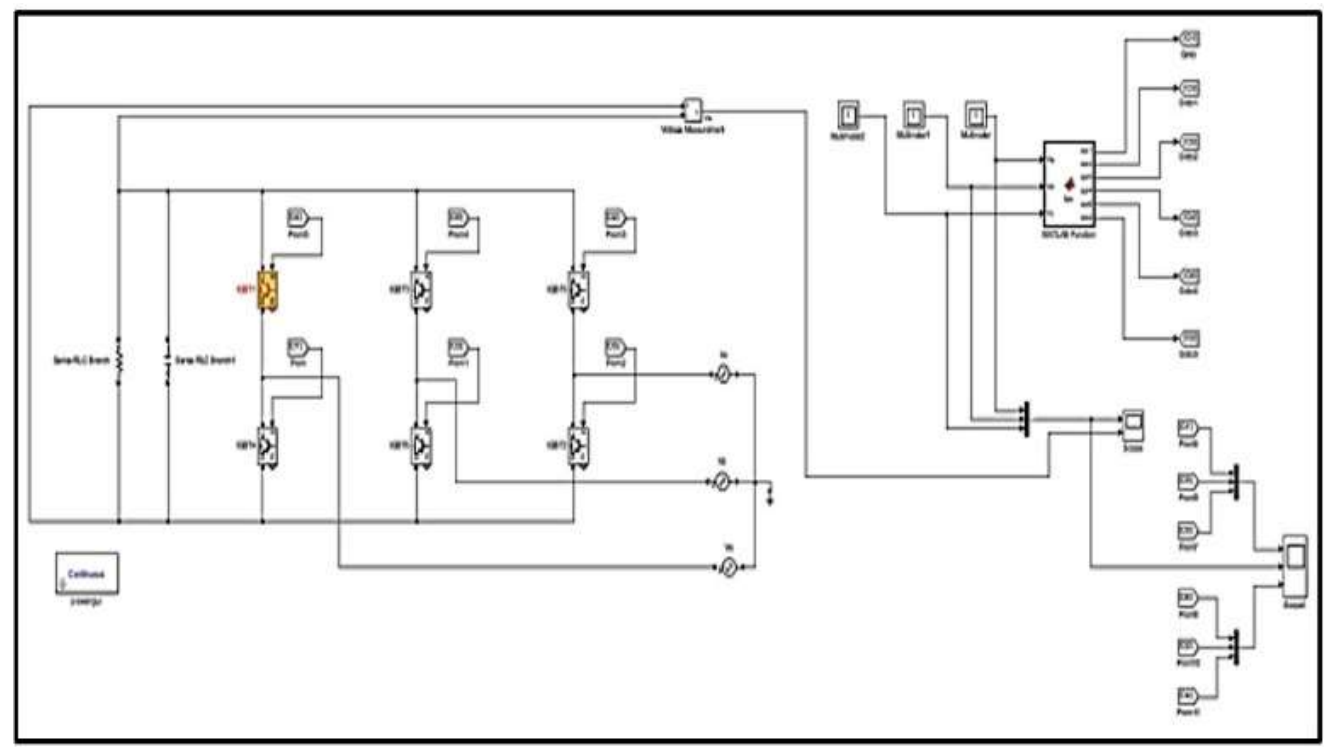

Figura 6: Esquema circuital en el rectificador

Tabla 2: Secuencia de Conmutación de los IGBTs.

\begin{tabular}{|l|r|r|r|r|r|r|}
\hline & $\boldsymbol{T}_{\mathbf{1}}$ & $\boldsymbol{T}_{\mathbf{2}}$ & $\boldsymbol{T}_{\mathbf{3}}$ & $\boldsymbol{T}_{\mathbf{4}}$ & $\boldsymbol{T}_{\mathbf{5}}$ & $\boldsymbol{T}_{\mathbf{6}}$ \\
\hline $\mathbf{1}$ & 1 & 0 & 0 & 0 & 0 & 1 \\
\hline $\mathbf{2}$ & 0 & 1 & 0 & 0 & 0 & 1 \\
\hline $\mathbf{3}$ & 0 & 1 & 0 & 1 & 0 & 0 \\
\hline $\mathbf{4}$ & 0 & 0 & 1 & 1 & 0 & 0 \\
\hline $\mathbf{5}$ & 0 & 0 & 1 & 0 & 1 & 0 \\
\hline $\mathbf{6}$ & 1 & 0 & 0 & 0 & 1 & 0 \\
\hline
\end{tabular}

Para la aplicación que se implementa, se desarrolla el código para obtener la tensión DC máxima, lo cual se consigue con $\alpha=0^{\circ}$. La evaluación simulada se hace con $\mathrm{L}=1 \mathrm{mH}, \mathrm{C}=2000 \mathrm{uF}, \mathrm{R}=1$ ohmios.

\section{Resultados}

En la figura 7 se muestra las corrientes trifásicas que circulan por cada fase de carga en conexión estrella, la optimización (minimización de la función de costo), se aplica a la corriente de fase, se minimiza el error entre el valor de referencia y el valor medido, diferencia que se busca reducir a cero en cada instante de muestreo.

Un indicador importante que evalúa el grado de distorsión generado por la conversión, es el THD. Estos resultados son presentados en las figuras 8, 9 y 10.

En la tabla 3 se presentan los resultados comparados correspondiente al THD entre el control MPC-FCS y el usando el control SVPWM.

En la figura 11 se muestra el resultado del convertidor trabajando como rectificador trifásico. 


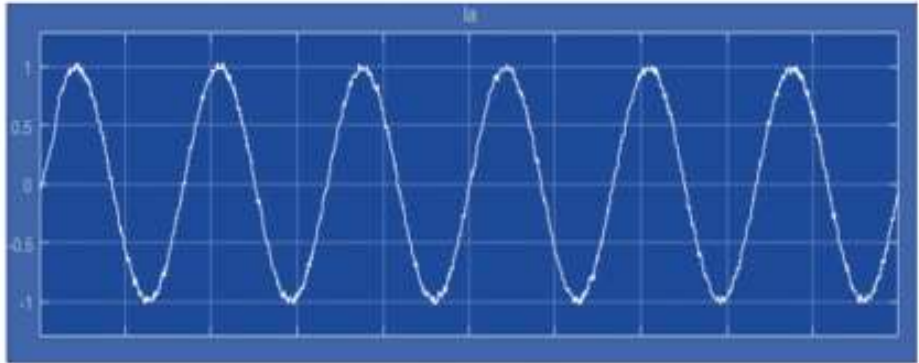

la

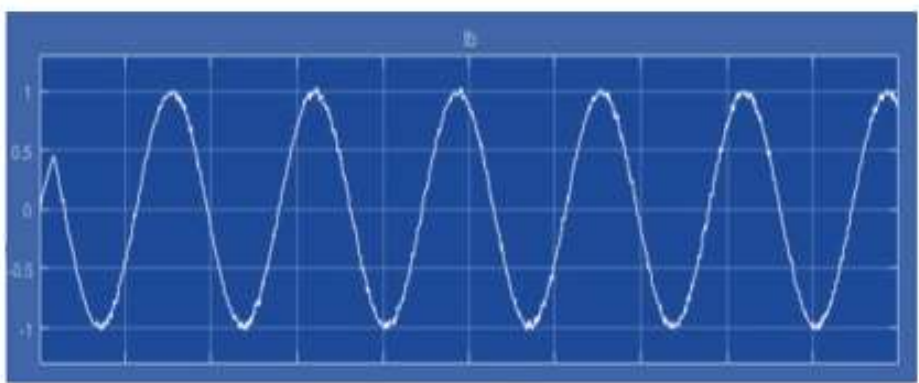

$\mathrm{Ib}$

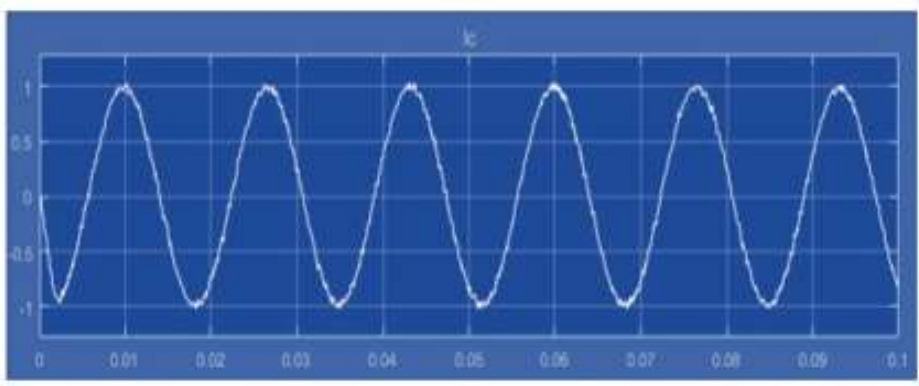

Ic

Figura 7: Corrientes eléctricas en cada fase de la carga en conexión estrella.

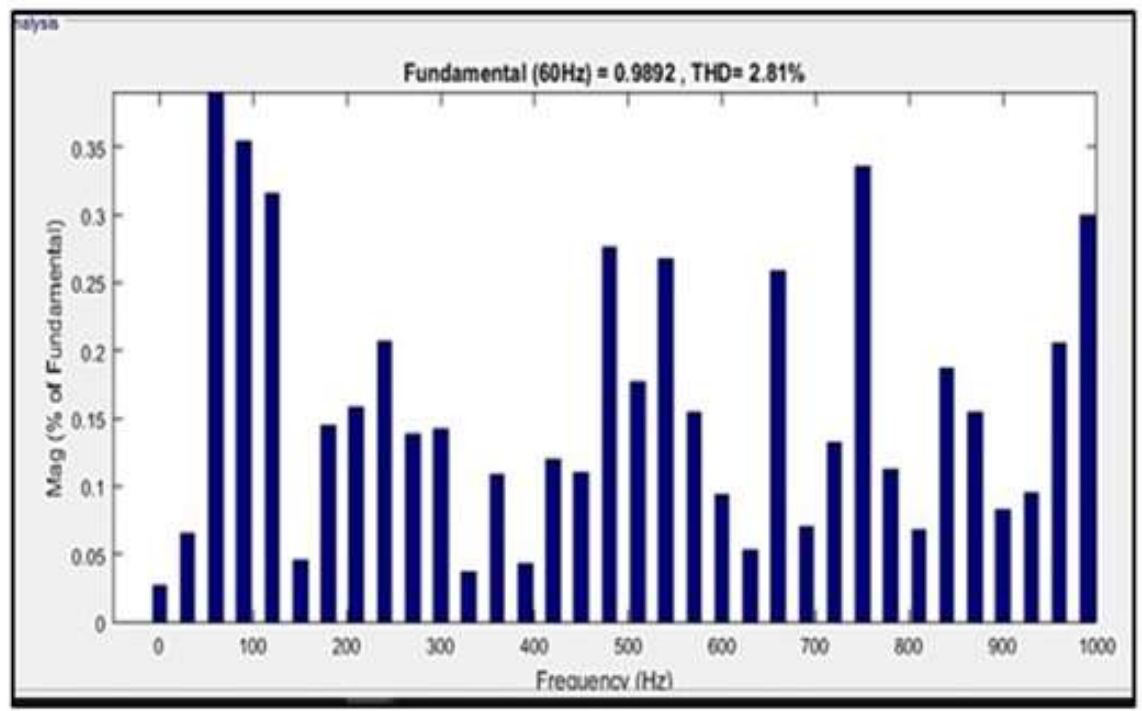

Figura 8: THD para la corriente en la carga correspondiente a la fase a (la). 


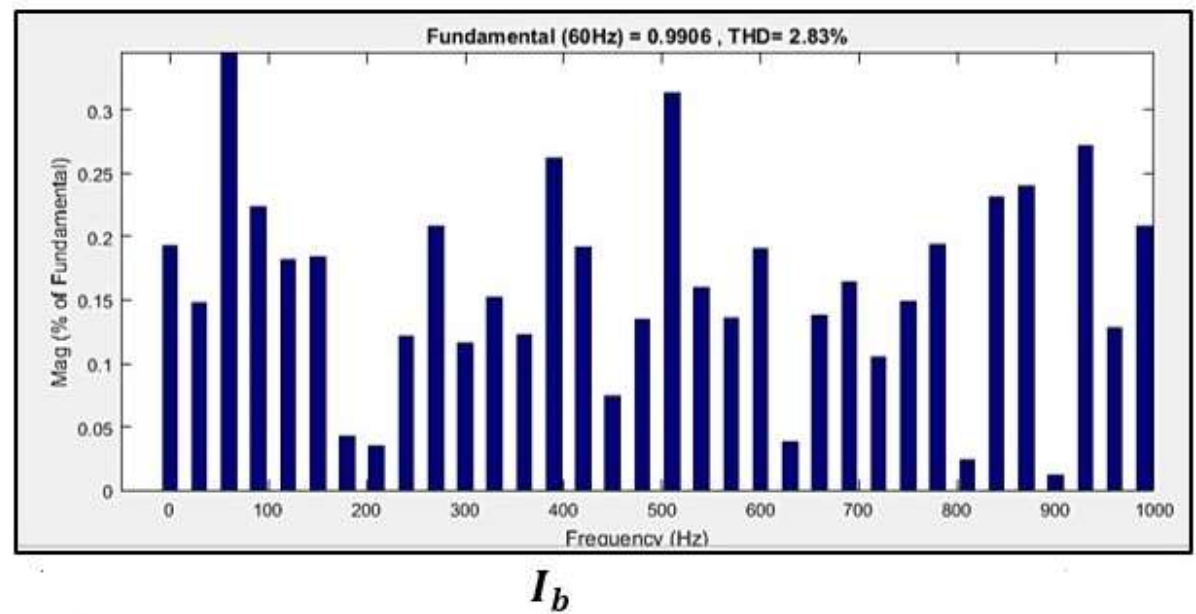

Figura 9: THD para la corriente en la carga correspondiente a la fase a ( $\left.\boldsymbol{I}_{b}\right)$.

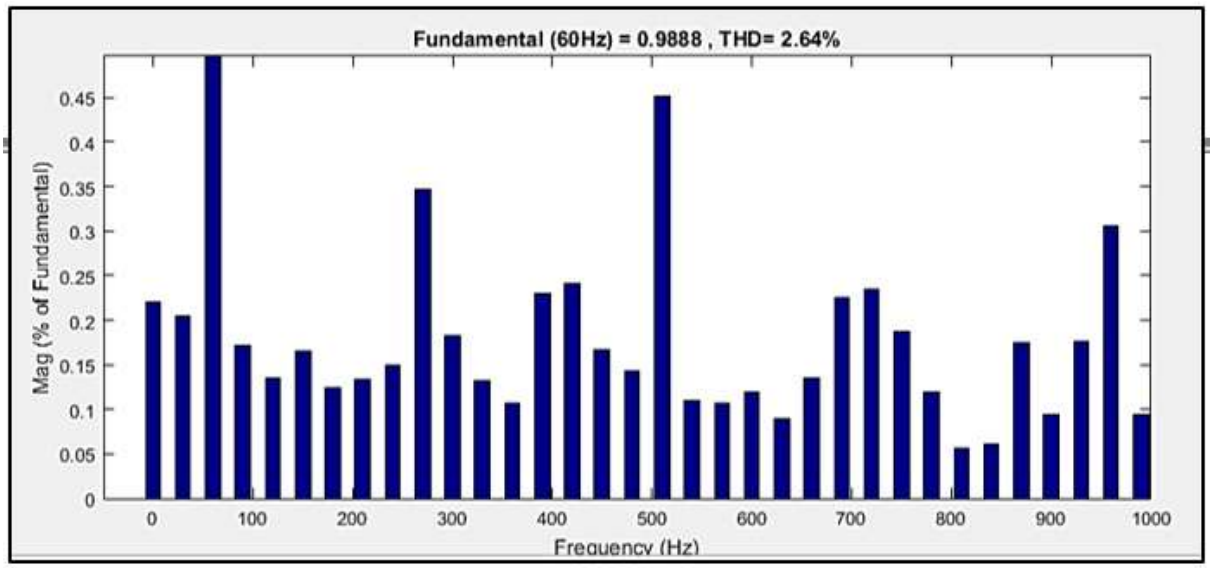

THD -Ic

Figura 10: THD para la corriente en la carga correspondiente a la fase a (Ic).

Tabla 3: THD en las corrientes, comparado entre MPC-FCS y SVPWM.

\begin{tabular}{|l|r|r|r|}
\hline & THD -la & THD-Ib & THD-IC \\
\hline MPC-FCS & $2.81 \%$ & $2.83 \%$ & $2.64 \%$ \\
\hline SVPWM & $4.48 \%$ & $4.47 \%$ & $4.47 \%$ \\
\hline
\end{tabular}

\section{Conclusiones}

El trabajo nos muestra la utilidad y ventajas de la utilización del control MPC-FCS al implementar el diseño de los sistemas de control de la etapa de potencia de convertidores electrónicos bidireccionales de potencia y energía eléctrica DC/AC, que además de tener un buen comportamiento en la conversión al tener una baja distorsión de armónicos (THD) el cual se encuentra en $2.7 \%$ en promedio, también queda en claro que a nivel de la unidad de control se le puede dar una robustez al sistema en cuanto a su mayor confiabilidad, además en el trabajo se aprecia que los conceptos sobre los cuales se desarrolla este tipo de control son relativamente muy simples.

Por otro lado la necesidad de tener convertidores que tengan una gran flexibilidad, como es el caso de este tipo de control, constituye un requerimiento que es muy necesario ahora y lo será más aún en los próximos años en que la generación distribuida y el énfasis de la generación con fuentes renovables de energía se haga más intensiva, de esto se concluye que trabajos en esta área de la ingeniería son importantes ya que cada solución es realmente muy específica pues esta debe satisfacer las demandas y utilizar los recursos propios de cada lugar específico en que 
se implemente una micro red y un sistema de generación distribuida.

La conversión bilateral de energía permite tener sistemas eficientes y compactos, aspecto de importancia en los sistemas energéticos, además se abre un campo para el desarrollo de sistemas de conversión de energía multidireccionales.

V1 DC

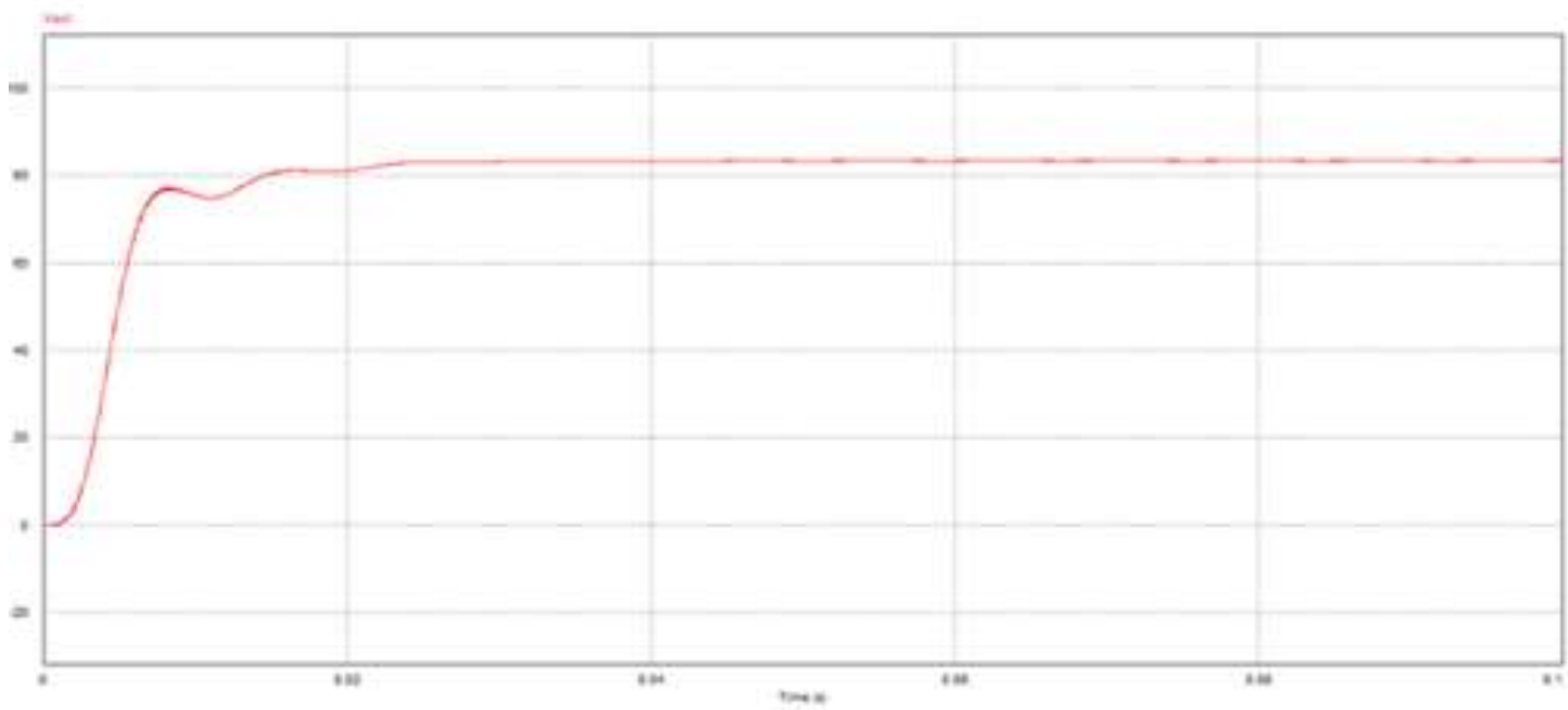

Figura 11: Tensión DC de salida en modo rectificador.

Se ha efectuado una comparación con técnicas bien conocidas de control de este tipo se sistemas de conversión de potencia, como es el caso del control SVPWM, obteniendo como resultado ventajas tanto en el comportamiento, así como en la mayor simplicidad de las soluciones.

\section{Referencias}

[1] M. Judewicz, J. Fischer, M. Herran, S. Gonzalez, D. Carrica, MPC con Modelo Predictivo basado en Observador de Estados, $23^{\circ}$ Congreso Argentino de Control Automático, (2012).

[2] M. Judewicz, J. Fischer, M. Herran, S. Gonzalez, D. Carrica, A Robust Model Predictive Control For Grid-Connected Voltage-Source Inverters, Latin America Transactions, IEEE 11 (2013) 27-33.

[3] C. Burdon, C. Montero, Basic Principles of MPC for Power Converters, IEEE Industrial Electronics Magazine 9 (2015) 31 - 43
[4] G. O. Riyadh, H.T. Rabee', Finite Control Set Model Predictive Current Control-MPC Based on Cost Function Optimization, with Current Limit Constraints for Four-Leg VSI, Iraq J. Electrical and Electronic Engineering 12 (2016) 43 - 53

[5] V. Monteiro, J. Ferreyra, Model Predictive Control Applied to an Improved Five-Level Bidirectional Converter, IEEE Transactions on Industrial Electronics 63 (2016) 58795890.

[6] P. Arul, V. Ramachandaramurthy, and R. Rajkumar, "Control strategies for a hybrid renewable energy system: A review," Renew. Sustain. Energy Rev. 42 (2015) 597-608.

[7] N. Hatziargyriou, Microgrids: architectures and control,( John Wiley \& Sons, 2014), pp. 4-30.

Email: itisza@gmail.com 